\title{
(in)Visibilité(s) du monstre
}

La question du monstre et du monstrueux fait florès depuis quelques années dans le milieu des études littéraires. Ces études interrogent le thème du monstre dans sa diachronie (histoire de la monstruosité), dans la création littéraire, dans le champ des représentations esthétiques, sociales, etc. Citons entre autres le numéro " Les représentations du monstrueux » paru dans la revue Imaginaire et inconscient (2004), Le Canari du Nazi: essais sur la monstruosité (Onfray 2013), la collection Qu'est-ce qu'un monstre? (Ibrahim 2015) ou la collection La littérature et ses monstres (Servane, Levacher et Prigent 2006).

Si le monstre pouvait à la Renaissance servir de signe, les monstres des siècles suivants sont redevables de la révolution scientifique, avec notamment l'essor de la tératologie. Au XIX ${ }^{\mathrm{e}}$ siècle, « vaste conservatoire des annales de la monstruosité » (Chauvaud 244), le monstre aux traits résolument humains pose la question de la dénaturation de l'être que les théories scientifiques de l'époque, notamment celle de l'hérédité, tentent de rationaliser en proposant une généalogie du monstrueux. Le monstre devient le miroir d'un pendant sombre de l'être humain repoussant les marges de l'ordinaire et du "normal". Qu'il s'agisse d'avertir, d'annoncer ou de montrer, le monstre peuple durablement l'imaginaire collectif.

Nous avons souhaité revenir sur la notion de "monstre" par le biais d'une réflexion bien connue de Corneille à l'égard de sa pièce, L'Illusion comique (1636). Dans la dédicace, le dramaturge l'a qualifiée d' " étrange monstre ». L'auteur faisait bien sûr référence à l'assemblage composite de sa pièce, mêlant différents genres dramatiques, et exhibait dès lors l'hybridité constitutive de son œuvre. Tout baroque que l'auteur puisse être, il n'était pas moins étonné de son œuvre, au point qu'elle lui paraisse inhabituelle, en rupture avec le cadre pourtant irrégulier de l'esthétique baroque. L'écart d'avec la norme se verrait donc redoublé à moins que le premier écart (la monstruosité) n'en soit pas un, ou du moins, ne soit plus (ou pas) pensé comme tel par Corneille. Bref, il y aurait des monstres qui n'étonnent pas (ou plus) car ceux-ci sont complètement banalisés dans l'imaginaire d'une époque. Ces monstres seraient en quelque sorte invisibles, au grand dam de l'étymologie du mot qui renvoie bien au champ du visuel observable (monstrare). C'est là aussi ce qu'illustre la chimère de l'illustration retenue pour la couverture de ce numéro. Du haut de NotreDame, la créature de pierre se dresse comme pour mieux observer la foule parisienne sans pour autant chercher à se dissimuler. Et pourquoi le ferait-elle ? Sa monstruosité est attendue (c'est une marque du style de la cathédrale depuis sa rénovation par Viollet-LeDuc dans les années 1850) et donc maintenant parfaitement anodine.

Ce faisant, de tels monstres perdent de leur pouvoir de fascination ou de peur selon la fonction que leur assignait Antoine Furetière dans son Dictionnaire universel (1702) : " Prodige qui est contre l'ordre de la nature, qu'on admire, ou qui fait peur », les monstres évoqués plus haut n'étonnent plus. Il semble que la fréquentation et l'habitude, bref le passage du temps, les aient dévitalisés aux yeux de ceux qui les côtoient. C'est peut-être une des fonctions de certains genres ou disciplines que de ranimer les monstres assoupis du quotidien. En effet, les chimères gothiques (re)prennent vie assez facilement sous l'œil de la caméra (voir le film Ghostbusters par exemple) mais aussi sous la mine d'architectes tel Viollet-le-Duc qui a fait ressurgir de la mémoire 
médiévale les 54 statues chimériques qui ornent Notre-Dame depuis sa restauration (Le Men). L'influence du roman gothique anglais et du romantisme semble bien ici indéniable. De même, le monstre de mots créé par Corneille n'est plus monstrueux. A tel point que les collégiens français y sont régulièrement confrontés pour s'interroger sur la raison ayant poussé Corneille à y voir un monstre. Depuis, on a admis, avec parfois bien du mal, dans le monde des lettres françaises, que l'hybridité générique n'était pas une tare.

Les contributions retenues pour ce numéro nous entraînent depuis la fin du XVIIIe siècle jusqu'à ce que l'on nomme de nos jours l'extrême contemporain en interrogeant les domaines littéraire et visuel.

Nous commençons donc au XVIII ${ }^{e}$ siècle avec « La déclinaison visuelle du monstre au XVIII siècle : Goya » de Juan Manuel Ibeas et Lydia Vásquez. Selon les auteurs, l'œuvre de Goya témoigne de l'évolution de la figure du monstre au cours du siècle des Lumières et semble s'inscrire dans le discours de Diderot et des encyclopédistes sur la question. Jusqu'alors marqué par les croyances religieuses et les théories naturalistes en cours, cet être effroyable, divin, merveilleux ou encore prodigieux, se prête davantage aux analyses scientifiques, étiologiques et tératologiques au XVIII ${ }^{e}$ siècle. Le monstre, autrefois perçu comme un Autre radical, tend à se normaliser et devenir un Même dans la mesure où il se fait omniprésent et tend à se rapprocher de la norme. Dans ses œuvres, Goya le décline sous plusieurs formes plus ou moins reconnaissables et visibles, de l'animal à l'homme, pour révéler la monstruosité de l'être humain.

Le monstre poursuit son processus d'invisibilisation au XIX ${ }^{\mathrm{e}}$ siècle en France. En effet, dans « Du "monstre humain" au "monstre-objet" : l'évolution du monstre (in)visible dans Notre Dame de Paris et A Rebours », Jade Patterson constate que la production de masse des monstres en littérature et dans les arts en général a tendance à banaliser leur apparence extraordinaire. Il s'agirait ainsi d'un passage du «monstre-humain visible », manifeste dans Notre Dame de Paris d'Hugo, au " monstre-objet invisible » caractéristique d'A Rebours de Huysmans. Un autre type de monstre, visible cette fois, se met également en place au cours de ce siècle: le « roman-monstre » dont les deux œuvres à l'étude sont des exemples. Ce dernier est marqué par l'excès et l'hybridité générique repoussant les limites des genres littéraires traditionnels.

Dans « Monstre et monstrueux dans l'œuvre de Georges Darien. Représentation et symbolique d'une chair meurtrie », Aurélien Lorig s'intéresse davantage au monstrueux des institutions du début du XIX ${ }^{\mathrm{e}}$-fin du $\mathrm{XX}^{\mathrm{e}}$ siècles pointées du doigt par Georges Darien dans des œuvres encore peu étudiées. Les figures monstrueuses qui peuplent l'univers littéraire de Darien sont ainsi le reflet, ou encore la production, du contexte social de l'époque. Ce miroir effrayant joue sur la dynamique visible-invisible auquel le lecteur doit participer afin de reconstruire le sens et d'interpréter la monstruosité de la société de l'époque.

Changement de siècle et de continent avec « Monstruosités et esthétiques du chaos dans les dramaturgies contemporaines africaines " de Sylvie Ngilla où l'auteure s'attache tout d'abord à remettre en question l'opposition classique sur la question de l'identité africaine entre traditionalistes et libertaires au théâtre. En passant en revue ce débat, elle situe rapidement le « 
nouveau théâtre africain » dès 1990 dans le contexte général de développement du théâtre en Afrique depuis les années 30 et se concentre sur deux traits fondamentaux : la monstruosité et le chaos. Le premier se manifeste sous forme d'hybridités multiples au sein d'une écriture qui détourne la syntaxe, les codes et les repères spatio-temporels pour mener au second, le chaos, compris comme une instabilité permanente menant à une identité fragmentée salutaire.

C'est également d'une redéfinition identitaire dans un contexte de renouveau postcolonial dans la littérature de l'océan Indien dont il est question dans « Aller vers la dissolution : hybridité, ambivalence et monstruosité chez l'écrivaine mauricienne Ananda Devi » de Markus Arnold. A travers son œuvre, la romancière mauricienne Ananda Devi propose un univers déstabilisant et opaque où les personnages sont " déréalisés », se rapprochant davantage du monstre que de l'humain. L'esthétique particulière de son œuvre dynamite les imaginaires et pose des questions de fond sur la complexité du monde contemporain.

Ce numéro est également l'occasion d'inaugurer une nouvelle section qui, nous l'espérons, provoquera maints débats et réflexions, avec l'essai critique de Milène Tournier, "Jean Barbin / François Bon : l'écriture monstrueuse, YouTube, l'édition du minuit de soi ». L'auteure prend pour objet d'étude les vidéos de François Bon mises en ligne sur YouTube où l'auteur apparaît sous les traits de Jean Barbin, être aux traits déformés dont les réflexions sur le monde qui l'entourent peuvent provoquer le malaise chez le spectateur.

Enfin, la section Varia, inaugurée dans le numéro 4.1, présente « Le Voyage pittoresque et historique de l'Istrie et de la Dalmatie de Joseph Lavallée: l'enchâssement textuel tardif des dessins de Louis François Cassas réalisés en $1782 »$. Dans cet article, Dragan Bogojević s'intéresse aux rapports entre texte et illustrations dans ce récit de voyage et à l'ambiguïté des rapports d'autorité entre le rédacteur (Lavallée) et l'illustrateur (Cassas). Lavallée (re)crée l'itinéraire de Cassas sur la base des illustrations de ce dernier mais réoriente dans le même temps la lecture qui peut en être faite par l'ajout de sa propre scénographie du voyage, ancrée dans la réflexion politique de la France postrévolutionnaire.

Pour leur soutien, nous tenons aussi à remercier Junia Barreto, Gilles Bonnet, Stéphanie Boulard, Chantal Brière, Aude Campmas, Adama Coulibaly, Sélom Gbanou, Pierre Hyppolite, Franck Laurent, Sandrine Lepors, Eric Lowen, Anne Malena, Catherine Mazaurie, Judith Miller, Max Poty, Myriam Roman, Baldine Saint-Girons, Philippe St Germain, Jean-Jacques Tatin-Gourier, Dany Toubiana, Patrice Terrone, Ritu Tyagi, Thanh-Vân Ton-That et Anthony Wall. 


\section{Bibliographie}

Chauvaud, Frédéric. " Les figures du monstre dans la seconde moitié du XIX siècle. » Ethnologie française 21.3 : 243-253.

www.jstor.org/stable/40989393. Corneille. L'illusion comique, 1636.

Furetière, Antoine. Dictionnaire universel, 1702.

Ibrahim, Annie, dir. Qu'est-ce qu'un monstre?. PUF, 2015.

Le Men, Ségolène. « De Notre-Dame de Paris au Stryge : l'invention d'une image. » Livraisons de l'histoire de l'architecture 20 (2010) : 49-74. DOI : 10.4000/1ha.25. Consulté le 03 avril 2018.

Onfray, Michel. Le Canari du Nazi: essais sur la monstruosité. J'ai Lu, 2013. « Les représentations du monstrueux. » Imaginaire et inconscient 13.1 (2004). https://www.cairn.info/revue-imaginaire-et-inconscient-2004-1.html. Consulté le 03 avril 2018.

Servane Daniel, Maëlle Levacher et Hélène Prigent, dir. La littérature et ses monstres, coll. « Horizons comparatistes. » Cécile Defaut éditeur, 2006.

\section{Image}

« Paris. Notre Dame Cathedral Tower. Chimeras and Man. » ca 1865 (détail), Cornell University Library. 